\title{
Flare productivity of newly-emerged paired and isolated solar active regions
}

\author{
S. Dalla ${ }^{1}$, L. Fletcher ${ }^{2}$, and N. A. Walton ${ }^{3}$ \\ 1 School of Physics and Astronomy, University of Manchester, PO Box 88, Manchester M60 1QD, UK \\ e-mail: s.dalla@manchester.ac.uk \\ 2 Department of Physics and Astronomy, University of Glasgow, Glasgow G12 8QQ, UK \\ 3 Institute of Astronomy, University of Cambridge, Cambridge CB3 OHA, UK
}

Received 26 January 2007 / Accepted 7 April 2007

\begin{abstract}
Aims. We investigate whether sunspot regions that emerge near existing ones are more flare productive than those that emerge isolated. Methods. We analyse a sample of 2115 new regions obtained from the USAF/Mount Wilson catalogue of sunspot regions. For our analysis we use AstroGrid, a Virtual Observatory developed in the UK, to build a series of workflows that perform queries to catalogues of solar regions and flares, and operations on the results of the queries. If a new region emerged through the solar surface within $12^{\circ}$ of a pre-existing one, we classify it as paired, otherwise as isolated.

Results. We find that paired regions are more flare productive than isolated ones, although this is a small effect. Here only soft X-ray flares of magnitude greater than C1.0 are considered, and flare productivity is characterised by the percentage of regions that produced at least one flare over 4 days since emergence, and by the average number of flares over the same period. For paired regions, we also consider the flare productivity of the nearby companion region and find that if a flare does happen within the pair, it will more likely take place in the companion region than in the newly-emerged one. Our results show that although emergence in proximity to another region can slightly increase the probability of a flare taking place, presumably by increasing the likelihood of magnetic reconnection and significant change in magnetic topology, this is not a large effect. It appears that intrinsic properties of the region are the key factor in determining whether or not it will produce flares, as opposed to interaction with pre-existing regions.
\end{abstract}

Key words. Sun: magnetic fields - Sun: flares - Sun: sunspots

\section{Introduction}

Solar flares are known to originate mostly within Active Regions. The accelerated particles and heliospheric magnetic disturbances produced by flares and the Coronal Mass Ejections (CMEs) that are frequently associated with them, are components of Space Weather. Identifying the characteristics of regions that produce medium to large flares and developing automated methods for prediction of flare activity are important aims of Space Weather research (Wheatland 2005). Flares are known to occur preferentially in regions which have a high degree of magnetic complexity and show rapid evolution and magnetic flux emergence. A high degree of magnetic complexity implies tangled or twisted field with ample free energy, and several studies, among them Künzel (1960), Zirin \& Liggett (1987), McIntosh (1990) and Sammis et al. (2000) demonstrate a relationship between the magnetic structure of a sunspot group and its chances of producing a flare. Most flares occur in compact groups of more than one polarity, in which the largest spot has a large asymmetric penumbra, with almost all flares above GOES X-class tending to occur in regions classified, albeit by subjective observers, as $\beta \gamma \delta$ - that is, in regions of high magnetic complexity. The emergence of new flux into a magnetised corona is the basic idea in the flare model of Heyvaerts et al. (1977), and many authors have investigated observationally the relationship between flux emergence and solar activity, where "activity" includes flares, filament eruptions and CMEs. In a study of non-Active Region (AR) filament eruptions (using coronagraph observations of CMEs as a proxy) Feynman \& Martin (1995) found that 19 eruptions of the 30 in their sample were preceded by the emergence of new flux, in the filament channel or within a few degrees of it, during the preceding 1 to 4 days. Jing et al. (2004) studying $\mathrm{H}_{\alpha}$ filament activity found that a similar fraction (54 of 80) of filament eruptions, including AR filaments, were associated with the emergence of new flux. Twenty of the 21 eruptions in active regions were associated with a flare. The emergence of new flux in or near pre-existing regions has recently been found by Schrijver et al. (2005) to be associated with significantly non-potential large-scale magnetic fields, and enhanced flaring activity.

In the Heyvaerts et al. (1977) model the flare activity takes place at a current sheet formed at the interface between new and pre-existing flux. They suggest that "the emerging flux usually provides energy for small flares and triggers additional release for large events" in field that is already pre-stressed, and that whether a large or small flare, or a surge or jet results, depends in the model on the "nature of the magnetic environment in which the emerging flux finds itself". One might therefore expect enhanced flaring when a new AR emerges in the neighbourhood of a pre-existing one, compared to if it emerges isolated into the weak field of the corona. Advanced MHD simulations exist of flux emergence into a pre-existing magnetic field, starting with the 2D simulations of Yokoyama \& Shibata (1995) which revealed the current-sheet and jet formation predicted by the Heyvaerts et al. model, however numerical techniques have not yet been applied to the case of flux emergence within, or in the close neighbourhood of, pre-existing non-potential field, 
the situation best described by the Heyvaerts et al. (1977) cartoons. That said, 3D modelling efforts are becoming very advanced, and include Galsgaard et al. (2005) and Archontis et al. (2005), who prescribe a uniform horizontal coronal field, and Isobe et al. (2005, 2006) whose coronal field is uniform and oblique. These authors identify fragmented current sheets, jetting behaviour and the formation of plasmoids as a consequence of the interaction. Fan \& Gibson $(2003,2004)$ and Gibson et al. (2004) emerge a twisted flux tube inside a coronal arcade having a potential field, and concentrate more on the overall shape of the current sheet, the topology of the system and the locations at which reconnection is likely to happen.

In this paper we will address the effect on an AR's flare productivity of the emergence of new magnetic flux in its neighbourhood. It is reasonable to expect that the emergence of new flux in the core of a large and complex active region will lead to the most energetic events. However, we are restricting our studies to the emergence of new flux in the close neighbourhood of a pre-existing companion AR. Part of the reason for this restriction is that such new emergence events are easily identified by new NOAA AR numbers, and our survey method, demonstrating grid computing techniques, currently requires these identifiers. However, by restricting our emergence criterion in this way we are also de facto focussing on the interaction between two initially unconnected regions, thereby investigating how much an external perturbation can promote flaring activity. Observationally a new AR emerging close to a pre-existing region does disturb its magnetic configuration. For example, early observations with TRACE (Schrijver et al. 1999) showed the distortion of the loops of an emerging AR towards a pre-existing one at a distance of some $10^{5} \mathrm{~km}$ (or $\sim 8^{\circ}$ in heliographic angle), followed by a period of surge and spray activity as magnetic connections (seen as EUV loops) formed. Bagalá et al. (2000) studied flaring between two adjacent regions, one newly-emerged and separated by about $200^{\prime \prime}$ from its previously-emerged companion. Longcope et al. (2005) follow the emergence of an AR within about $200^{\prime \prime}$ of a pre-existing companion region, which also showed clear evidence of reconnection between the two regions, though not associated with significant flare activity; the GOES level at the time of maximum reconnection is C2.8. So the influence of one active region certainly extends beyond its photospheric footprint, but by how much, and to what effect?

We will investigate (i) whether the emergence of an AR in close proximity to another one will make it or its companion more flare-productive compared to an AR that emerges isolated and (ii) whether the flaring occurs predominantly in the newly-emerged or in the older companion region. This study requires the systematic search and comparison of several catalogue databases, a task which is accomplished using the AstroGrid programming environment. AstroGrid, the UK's contribution to a global Virtual Observatory (VO), allows users to access several Astronomy and Solar System catalogues and datasets, and to develop workflows that operate on data. One of the main aims of VOs is to provide standardised access to data from diverse sources to allow easy comparison and integration of diverse datasets. Our study presents an example of how the AstroGrid workflow environment allows a user to retrieve information from multiple catalogues, operate on this information and analyse the results in a way that is independent of the specific catalogues under consideration.

In Sect. 2 we describe the steps required to investigate flare productivity in paired and isolated regions and the scientific assumptions we made. Our results are presented in Sect. 3 and a discussion is given in Sect. 4.

\section{Science workflows}

Three main steps are needed to establish the flare productivity of newly-emerged paired and isolated regions. Each step is coded as a separate AstroGrid workflow.

The first step consists of generating a catalogue of newlyemerged regions, by identifying emergence on the visible disk of the Sun (Sect. 2.1). In the second step, we evaluate whether each of our newly-emerged regions did so in an isolated location, or in near proximity to another pre-existing region (Sect. 2.2). Finally, we examine the flare productivity of the newly-emerged and preexisting regions by cross correlating our region catalogue with the GOES soft X-ray flare list (Sect. 2.3).

Section 2.4 briefly describes the AstroGrid workflow environment and the VOTable format that was used for the catalogues of solar regions and flares.

\subsection{Generating a catalogue of newly-emerged regions}

Active regions on the Sun are routinely identified and catalogued. They are regions of strong magnetic fields, that appear bright in $\mathrm{H}_{\alpha}$ and X-rays, and in which activity such as flares tends to take place. The NOAA Space Environment Center (http://www.sec.noaa.gov/) assignes an Active Region number to solar regions. An Active Region number is assigned to a region that satisfies one of the following criteria: (1) the region has been reported to have a sunspot group with first digit of its Modified Zurich Classification of C, D, E, F or H; (2) two or more reports confirm the presence of a Modified Zurich class A or B sunspot group; (3) the region produces a solar flare; or (4) the region is bright in $\mathrm{H}_{\alpha}$ and exceeds 5 heliographic degrees in either latitude or longitude.

In our study, we have made use of two catalogues: the NOAA Solar Region Summary and the USAF/Mount Wilson catalogue of sunspot regions. Both are published as part of the NOAA Solar Geophysical Data. The two catalogues are not completely independent since the SRS is compiled by making use of USAF/Mount Wilson observations, among others.

We started our analysis by using the Solar Region Summary (SRS), whose entries from 1996 onwards can be accessed via the AstroGrid interface to the EGSO Solar Event Catalogue (Bentley \& EGSO Consortium 2002). The SRS is a summary report detailing which Active Regions are observed on disk every day, and their parameters, including location and magnetic classification. The list combines information from several observatories. In addition to sunspot regions, this catalogue also lists $\mathrm{H}_{\alpha}$ plages without spots.

The USAF/Mount Wilson (USAF/MWL) catalogue ${ }^{1}$ provides a list of sunspot regions and their parameters, observed by the USAF solar observatories and the Mount Wilson observatory. Unlike the SRS, it is not a summary, but a collection of all reports related to sunspot regions, i.e. if a region has been observed by say 4 observatories on a given day, the catalogue will contain all 4 entries. The USAF/MWL catalogue contains records starting in December 1981, hence provides a much larger sample of regions than the SRS list provided by the EGSO Solar Event Catalogue.

Information common to the two catalogues are the NOAA Active Region Number (nar) corresponding to the region, the time of observation and the corresponding heliographic latitude and longitude measured with respect to the Central Meridian

\footnotetext{
1 ftp://ftp.ngdc.noaa.gov/STP/SOLAR_DATA/ SUNSPOT_REGIONS/USAF_MWL/
} 


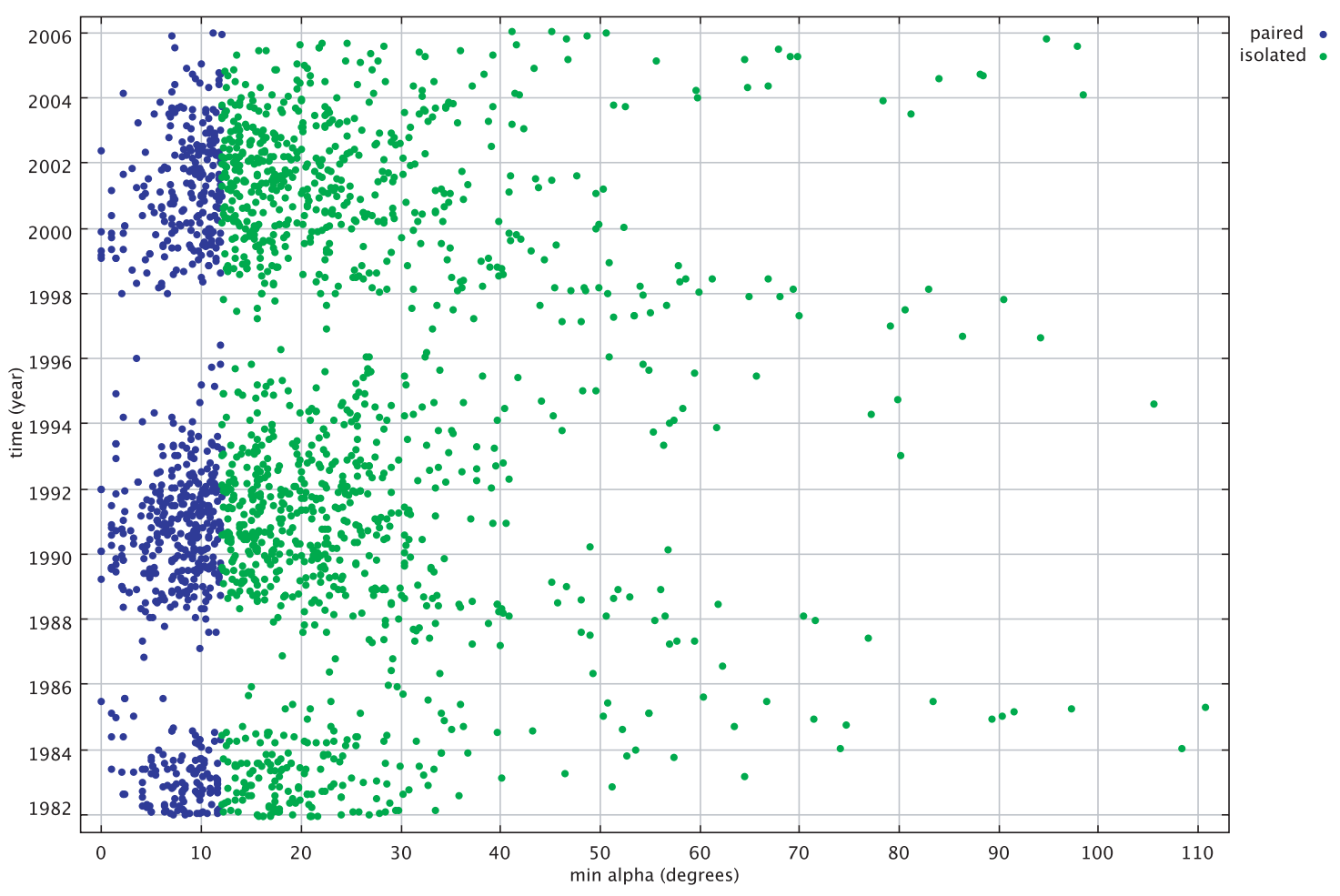

Fig. 1. Plot of time of NER emergence versus the great circle angle (in degrees) to the nearest sunspot region on disk on that day. Green symbols indicate isolated NERs, and blue symbols paired ones, with the cutoff angle $\alpha_{\text {paired }}=12^{\circ}$.

(referred to as longitude in the following), its Carrington longitude, Zurich classification, area, number of sunspots, longitudinal extent and Mt. Wilson magnetic classification.

Initially, we developed workflows that used the SRS as the region catalogue. We then repeated the analysis using the USAF/MWL catalogue, to improve our statistics: the results reported in this paper are from the USAF/MWL analysis. Repeating the analysis for a second separate catalogue was made easier by having coded it as AstroGrid workflows, that operate on VOTables and do not require a strict specification of the catalogue format.

Our first step was to identify Newly-Emerged Regions (NERs), that emerged on the visible side of the Sun. To this end, we developed a workflow that identifies the first entry in time associated with each NOAA region number. A VOTable of these entries was produced and the value $\lambda$ of the region longitude at 12:00 UT on the day on which it emerged calculated. This table listed information on 6862 separate sunspot regions identified from the USAF/MWL catalogue.

The value of the longitude $\lambda$ is then used to identify NERs as opposed to regions that rotated into view. Here the longitude is with respect to an Earth observer, with $\lambda=0$ corresponding to the Earth-Sun line, negative values to eastern longitudes and positive ones to western longitudes. To exclude regions that rotated into view, we excluded all regions with value of $\lambda$ to the east of $-60^{\circ}$, and classified all other regions, first observed to the west of $-60^{\circ}$ as NERs. For the time period under study, we obtained an initial list of 3265 NERs.

Although our cutoff value of $-60^{\circ}$ for identifying NERs is somewhat conservative, being more than $28^{\circ}$ (the longitude interval "travelled" by a sunspot region over two days) away from the east limb, this value ensures that regions that rotated into view but were included in the catalogue late, are not part of our sample.

\subsection{Establishing if the solar region emerged on disk isolated or paired}

The next step consists of establishing, for each NER in the list obtained from the workflow of Sect. 2.1, whether it emerged isolated or in near proximity to another, pre-existing, region.

As a proxy for the distance between 2 regions, we calculate the Great Circle angle $\alpha$ between them, that is the angle to the centre of the Sun subtended by the 2 regions. The actual distance can be obtained by multiplying $\alpha$ by the solar radius. The calculation uses the values of longitude and latitude of the geometric centers of the two groups, as given in the sunspot region catalogue.

The AstroGrid workflow we developed takes each NER and works out where all other regions were located on the day of its emergence. It then calculates the great circle angles between the NER and all other regions. If one of these angles is smaller than a cutoff value $\alpha_{\text {paired }}$, then the NER is classified as "paired", if all distances are larger than $\alpha_{\text {paired }}$, it is classified as "isolated". Note that in a minority of cases the number of regions within $\alpha_{\text {paired }}$ of the NER is greater than 1, so this is not strictly speaking a "pair".

Figure 1 shows a plot of the time at which each NER was observed, versus its distance (expressed in terms of the Great Circle angle $\alpha$ ) to its nearest neighbour on the day of emergence. The plot shows a solar cycle dependence, as expected since near solar minimum the average number of regions on disk is small and the nearest neighbour to a NER is in many cases at large angular distances. Figure 2 shows the variation of the number of regions on disk versus time, for each time at which a NER was first observed.

For our analysis we choose a cutoff angle for the definition of paired vs. isolated given by $\alpha_{\text {paired }}=12^{\circ}$ (corresponding to a separation distance of $1.46 \times 10^{5} \mathrm{~km}$ (or 200" at disc-centre)). This is the same separation as in the Longcope et al. (2005) study. 


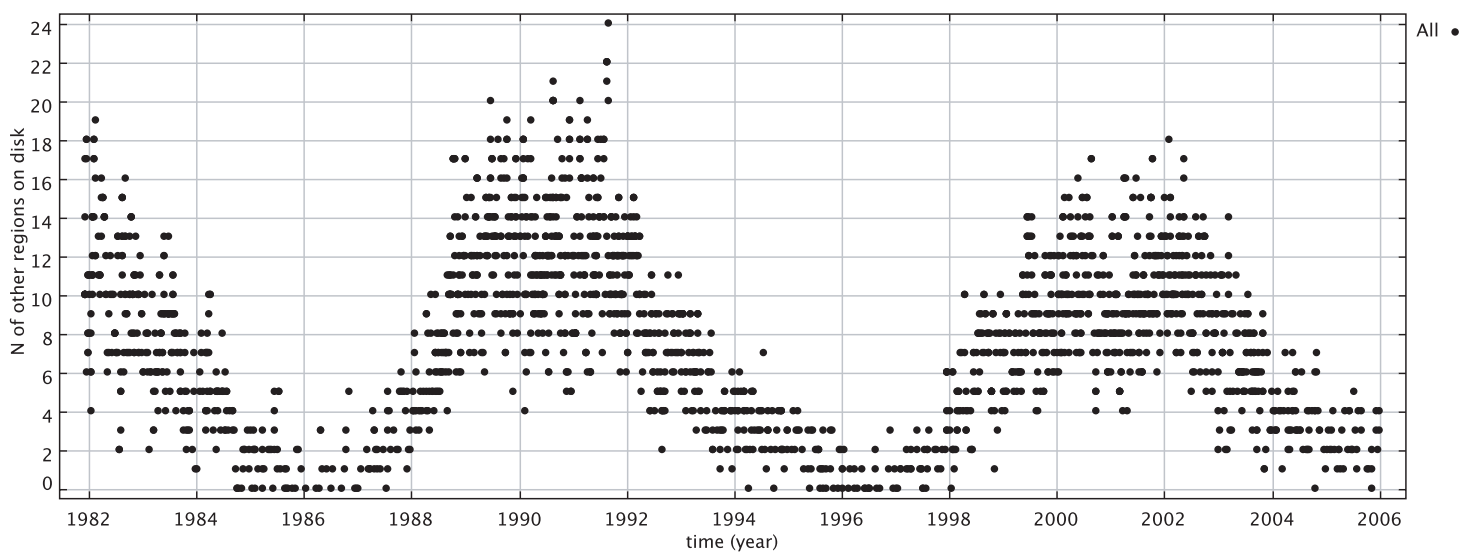

Fig. 2. Plot of the number of other regions present on disk, for each day in which a new region emerged.

With the choice $\alpha_{\text {paired }}=12^{\circ}$, and the further restriction that the regions should be visible for the time interval we use for counting flares (see Sect. 2.3), we obtain a sample of 675 paired NERs, and 1440 isolated ones.

\subsection{Obtaining flare productivity}

The next step in our analysis consisted of evaluating how many flares were produced by the NERs and, where applicable, their companions. Here we counted GOES soft X-ray flares above a cutoff magnitude of C1.0, to ensure good flare visibility. Our cutoff magnitude is smaller than the flaring activity recorded by Longcope et al. (2005) in their study of the interaction between neighbouring regions.

We developed a workflow that queries the EGSO Solar Event Catalogue to retrieve a table of GOES soft X-ray flares above C1.0. Among the flare characteristics in the GOES flare list is the NOAA Active Region Number associated with the flare, in those cases where an association could be made. Our workflow goes through our catalogue of NERs and makes use of the Active Region Number, to establish how many flares were produced by each region. This method is adequate for flares of medium to large magnitude, for which the AR number is recorded in a majority of cases. It was pointed out by Wheatland (2001) that approximately $60 \%$ of flares of magnitude greater than $\mathrm{C} 1.0$ are associated with an AR number in the GOES catalogue; in that work it was also found that a large number of flares are undetected by GOES due to them occuring in the declining, but still strong, gradual phase emission of a large event, above which it was difficult to discern them.

We obtained information on flares associated with each region for a period of 4 days since their emergence. To make sure that the regions we considered were visible from Earth for at least 4 days, we discarded from our list of NERs those that emerged in locations near the western limb and also excluded regions with short lifetime. This reduced the number of NERs in our list from 3265 to 2115 . Our workflow adds the number of flares produced over 4 days to our table of NERs.

Flare productivity can be defined in a number ways. We use as indicators of flare productivity the percentage $P$ of regions that produced at least one flare over the 4-day period considered, and also the average number of flares $F$ produced (in units of flares/(4 days)) over the same time range.

We study the flare productivity of newly-emerged regions, and for those that were paired, we also analyse flare productivity of the companion(s) for 4 days after the emergence of the NER in their vicinity, provided that they were visible during this time.

In addition, we also define a sample of old regions, by extracting from our initial list of regions those with values of longitude on the first day of observation between $-81^{\circ}$ and $-72^{\circ}$. Although this sample contains some NERs, it is dominated by old regions that rotated into view. When looking at old regions, we did not attempt to identify returning ones, i.e. a region that rotated into view twice will have two entries in our list.

\subsection{The Astrogrid workflow environment}

AstroGrid (http://wwW.astrogrid.org) released its version 2007.1 in January 2007 and is available to scientists as a tool for their investigations. The Workflow environment allows AstroGrid users to send queries to data centers and operate on the results of queries, to develop complex workflows. Results of queries are returned in VOTable format ${ }^{2}$, an XML format developed for the exchange of data within a Virtual Observatory (VO) context.

Queries to data centers need to be formulated in ADQL (Astronomical Data Query Language), which is a subset of SQL (Standard Query Language). Queries are built using a query builder, in which the metadata associated with each dataset or catalogue can be visualised, and be queried upon.

Queries are executed as workflow steps, and VOTables resulting from queries can be manipulated by using the Java STIL library (Starlink Tables Infrastructure Library, Taylor 2005) in the workflow environment. This allows users to extract columns of the table by specifying their name, calculate new columns from existing ones using logical and mathematical operations and add them to the table, compare columns of two VOTables etc. The workflows described in Sects. 2.1-2.3 make extensive use of this capability, which is independent of the particular table one is operating on.

The workflows described in Sects. 2.1-2.3 were run in succession and each stage added more information to the VOTable of solar regions. The final VOTable, containing distance and flare productivity information was opened using TOPCAT ${ }^{3}$ (Taylor 2005) and the results visualised and analysed.

\footnotetext{
2 The VOTable format is described at: http://www . ivoa.net/Documents

3 TOPCAT is available at: http://www.starlink.ac.uk/topcat/
} 


\section{Results}

We ran the three workflows described in Sects. 2.1-2.3 for USAF/MWL sunspot regions catalogued between 1 December 1981 and 31 December 2005, i.e. covering 24 years of data and spanning more than 2 solar cycles.

Table 1 shows the flare productivity parameters $P$ and $F$ (as defined in Sect. 2.3) for several groups of sunspot regions, with the corresponding Poisson errors. The groups we consider are: all NERs, paired ones, isolated ones, the companions of the paired NERs, and a group of old regions that rotated into view.

From Table 1 we can see that paired NERs are more likely to produce flares than isolated ones, although the difference between the two populations is small. In general, NERs have low flare productivity compared to the companions and old regions. Companion regions are not significantly more flare productive than old regions that rotated into view.

Table 1 allows us to compare the flare productivity of the two types of regions forming a pair: new regions in a pair (NERs paired) have a value of $P=24.3 \% \pm 1.9 \%$ while companions have $P=39.3 \% \pm 2.4 \%$. Therefore, within a pair, a flare is more likely to take place in the pre-existing companion region than in the NER.

The average number of flares $F$ during the 4-day period of observation displays a similar trend to the percentage $P$ of regions with flares.

It should be noted that the initial number of companion regions was larger than 676, however several had to be discarded because they were not observable over the entire 4 day period since the emergence of the NER. In addition, the 676 companion regions are not necessarily 676 distinct regions.

Table 1 was obtained by choosing a cutoff value between the paired and isolated populations of $\alpha_{\text {paired }}=12^{\circ}$. We also repeated the analysis for a value of $\alpha_{\text {paired }}=10^{\circ}$, for which the number of paired NERs becomes 510 . Since the sample of paired regions is smaller in this case, and as flaring over a period of 4 days is fairly rare (as shown in Table 1), the error on the value of $P$ for paired NERs becomes larger than for the case $\alpha_{\text {paired }}=12^{\circ}$, and the difference in the value of $P$ for paired versus isolated NERs is not statistically significant.

To understand the low flare productivity of NERs, we analysed the magnetic complexity of our samples of sunspot regions. The Mt. Wilson magnetic classification of sunspot regions is given in the region catalogues we analysed. For each region we identified the classification of highest magnetic complexity assigned to it, according to the following ordering of magnetic complexity of a sunspot region:

$[\alpha, \beta, \beta \gamma, \beta \delta, \gamma \delta, \beta \gamma \delta]=[1,2,3,4,5,6]$

where on the r.h.s. is the magnetic complexity index associated with each magnetic classification. For each region in our sample, our workflow extracts all its magnetic classifications for its entire lifetime on disk, and assigns a "maximum magnetic classification" given by the classification of highest magnetic complexity it reached according to the above scale.

Table 2 gives the the number of regions belonging to each of the maximum magnetic classification categories. The catalogue used for this analysis is the SRS list (containing 2880 regions), because it provides a more uniform catalogue of magnetic classifications than the USAF/MWL one. Table 2 shows that NERs tend to be magnetically "simple" regions, as can be expected since they are young regions. We found no significant difference in magnetic complexity between paired and isolated NERs.
Table 1. Flare productivity of paired NERs, isolated ones, the companions of paired regions, and old sunspot regions. Here $P$ is the percentage of regions with flares and $F$ is the average number of flares in the sample, over a period of 4 days and considering only flares greater than C1.0. The cutoff angle used in the definition of paired vs. isolated regions is $\alpha_{\text {paired }}=12^{\circ}$.

\begin{tabular}{lccc}
\hline \hline Subset & $n$ of regions & $P(\%)$ & $F$ (flares/4 days) \\
\hline NERs & 2115 & $21.7 \pm 1.0$ & $0.639 \pm 0.017$ \\
NERs paired & 675 & $24.3 \pm 1.9$ & $0.696 \pm 0.032$ \\
NERs isolated & 1440 & $20.5 \pm 1.1$ & $0.613 \pm 0.020$ \\
companions & 676 & $39.3 \pm 2.4$ & $1.745 \pm 0.051$ \\
old regions & 1516 & $39.7 \pm 1.6$ & $1.689 \pm 0.033$ \\
\hline
\end{tabular}

As was pointed out by Sammis et al. (2000) the magnetic complexity of a region is very important in determining whether or not it will produce any flares. Hence the low flare productivity of NERs can be broadly understood in terms of their low magnetic complexity. A more detailed discussion of the properties of a single region that make it prone to flaring can be found in Leka \& Barnes (2007).

As additional information to the results of Table 1 and Table 2, Table 3 shows the flare productivity of sunspot regions depending on their maximum magnetic classification, where flares $>\mathrm{C} 1.0$ and $>\mathrm{C} 5.0$, produced during the entire time that a given region was observed, are considered.

Of the 2880 regions in the SRS sample, only 584 (20\%) produced any flares above C5.0, and $1111(38 \%)$ had flares above C1.0. Only $0.7 \%$ of regions with maximum magnetic classification $\alpha$ in our population produced any flares above C1.0. The proportion of flare producing regions increases with magnetic classification up to $100 \%$ for regions with maximum classification $\beta \gamma \delta$, for flares above $\mathrm{C} 1.0$.

\section{Discussion}

From this study we have found that (i) newly-emerged paired regions are more likely to produce at least one flare for a period of four days after emergence, than isolated NERs; (ii) the average number of flares for paired regions compared to isolated ones is larger; (iii) the majority of the flares occurring in a pair do so in the previously-emerged companion region.

However, the difference in flare productivity between paired and isolated regions is a small effect. This suggests that the external magnetic environment is a secondary factor in a region's flaring history: the flare productivity depends primarily on some intrinsic property of the region. A fourth result, one which we did not initially seek, is that (iv) the maximum magnetic complexity reached by NERs is less than for the companion regions. Bagalá et al. (2000) in their study of a single region pair also noted that the companion AR was more complex and more flare-productive than the emerging one; our finding appears to generalise this.

The model of Heyvaerts et al. (1977) is partly supported by our findings; paired regions have enhanced flaring rates compared to isolated ones, which could suggest that interaction between NE and pre-existing flux plays a role in flare triggering. The majority of the flaring is also confined to the previouslyemerged and generally more magnetically-complex AR. This is in accordance with Heyvaerts et al.'s suggestion that the flux emergence may act to trigger release of free energy in the region into which it emerges, rather than the flare being powered by the magnetic free energy of the emerging region itself.

However, our sample is dominated by values of the minimum separation distance between NERs and their companions that are 
Table 2. Percentage of regions with given maximum magnetic classification. Data are from the analysis using the SRS catalogue.

\begin{tabular}{lcccccc}
\hline \hline Subset & number of regions & $\alpha(\%)$ & $\beta(\%)$ & $\beta \gamma(\%)$ & $\beta \delta(\%)$ & $\beta \gamma \delta(\%)$ \\
\hline All & 2880 & 10 & 73 & 11 & 0.8 & 5.2 \\
NERs & 1449 & 10 & 82 & 6 & 0.3 & 1.7 \\
companions & 468 & 6 & 73 & 13 & 0.6 & 7 \\
old regions & 1003 & 8 & 61 & 18 & 1.6 & 11 \\
\hline
\end{tabular}

Table 3. Percentage of regions with flares, depending on maximum magnetic classification. Data from the SRS catalogue (2880 regions).

\begin{tabular}{lcccccc}
\hline \hline Cutoff & All $(\%)$ & $\alpha(\%)$ & $\beta(\%)$ & $\beta \gamma(\%)$ & $\beta \delta(\%)$ & $\beta \gamma \delta(\%)$ \\
\hline Flares > C1 & 38 & 5 & 30 & 90 & 87 & 100 \\
Flares > C5 & 20 & 0.7 & 11 & 63 & 71 & 89 \\
\hline
\end{tabular}

not particularly small. We are not dealing with NERs that bubble up right at the core of an older region, primarily because such emergences are not given new NOAA active region numbers and so cannot be identified in our search.

As we have found, the perturbation due to quite distant emergences is limited. This is consistent with the majority of the free energy in a region being concentrated in the core, perhaps within a few $1000 \mathrm{~km}$ of the neutral line (as vector magnetic field observations lead us to believe) so that flux emergence at a few tens of thousands of $\mathrm{km}$ has little influence upon it, and does not lead to energetically significant flares. The flare energy will also depend on whether flux emerges at the location and with the appropriate orientation to make accessible a new topological state with significantly lower energy.

Our analysis shows that the magnetic complexity leading to copious flare production takes more than 4 days to develop. This implies a timescale much longer than the $\sim 1$ day observed for helicity to build up and reach a plateu in an active region (Pevtsov et al. 2003), but comparable to the $\sim 6$ days found theoretically for the relaxation to equilibrium of the twist transferred between a sub-photospheric flux tube and an emerging active region (Longcope \& Welsch 2000).

Our analysis would ideally be extended in future to incorporate regions in which new flux emerges in the core of a solar region. This kind of study would require image processing capabilities to identify such occurrences.

Acknowledgements. This research has made use of data obtained using, or software provided by, the UK's AstroGrid Virtual Observatory Project, which is funded by the Science and Technology Facilities Council and through the EU's Framework 6 programme. L.F. acknowledges the support of NASA grant NNG05GG17G.

\section{References}

Archontis, V., Moreno-Insertis, F., Galsgaard, K., \& Hood, A. W. 2005, ApJ, 635, 1299

Bagalá, L. G., Mandrini, C. H., Rovira, M. G., \& Démoulin, P. 2000, A\&A, 363, 779

Bentley, R. D., \& EGSO Consortium. 2002, in Solar Variability: From Core to Outer Frontiers, ed. A. Wilson, ESA SP-506, 923

Feynman, J., \& Martin, S. F. 1995, J. Geophys. Res., 100, 3355

Galsgaard, K., Moreno-Insertis, F., Archontis, V., \& Hood, A. 2005, ApJ, 618, L153

Gibson, S. E., Fan, Y., Mandrini, C., Fisher, G., \& Demoulin, P. 2004, ApJ, 617, 600

Heyvaerts, J., Priest, E. R., \& Rust, D. M. 1977, ApJ, 216, 123

Jing, J., Yurchyshyn, V. B., Yang, G., Xu, Y., \& Wang, H. 2004, ApJ, 614, 1054

Künzel, H. 1960, Astron. Nachr., 285, 271

Leka, K. D., \& Barnes, G. 2007, ApJ, 656, 1173

Longcope, D. W., \& Welsch, B. T. 2000, ApJ, 545, 1089

Longcope, D. W., McKenzie, D. E., Cirtain, J., \& Scott, J. 2005, ApJ, 630, 596

McIntosh, P. S. 1990, Sol. Phys., 125, 251

Pevtsov, A. A., Maleev, V. M., \& Longcope, D. W. 2003, ApJ, 593, 1217

Sammis, I., Tang, F., \& Zirin, H. 2000, ApJ, 540, 583

Schrijver, C. J., Title, A. M., Berger, T. E., et al. 1999, Sol. Phys., 187, 261

Schrijver, C. J., DeRosa, M. L., Title, A. M., \& Metcalf, T. R. 2005, ApJ, 628, 501

Taylor, M. B. 2005, in Astronomical Data Analysis Software and Systems XIV, ed. P. Shopbell, M. Britton, \& R. Ebert, ASP Conf. Ser., 347, 29

Wheatland, M. S. 2001, Sol. Phys., 203, 87

Wheatland, M. S. 2005, Space Weather, 3, 131

Yokoyama, T., \& Shibata, K. 1995, Nature, 375, 42

Zirin, H., \& Liggett, M. A. 1987, Sol. Phys., 113, 267 